\title{
EDITORIAL
}

\section{Compilation of online resources of relevance to 'Spinraza and advanced therapies: a stakeholder special' issue of Gene}

\section{Therapy}

Gene Therapy (2017) 24, 599; doi:10.1038/gt.2017.61

Spinal muscular atrophy (SMA) is the leading genetic cause of death in childhood. Spinraza (nusinersen) is the first marketed therapy for SMA, approved in the USA in December 2016 and in Europe in June 2017. To highlight the approval of Spinraza, Gene Therapy is publishing 'Spinraza and Advanced Therapies: a stakeholder special'. The purpose of the current compilation is to provide a set of online materials of relevance to the special issue. The weblinks include resources from governments, charitable associations, scientific societies and commercial information providers. The list is not exhaustive but rather aims to provide a starting point to navigate this field. Neither the authors nor Gene Therapy are responsible for the content of these external websites. The weblinks are correct at the time of going to press. Please paste the corresponding full hyperlink into your browser address bar if clicking on the link does not lead to the correct web page.

- Nusinersen mechanism (Youreka Science in YouTube): https:// www.youtube.com/watch?v=wrN-BRrzZ5E

- NIH Genetics Home Reference database entry on SMA: https:// ghr.nlm.nih.gov/condition/spinal-muscular-atrophy\#

- Online Inheritance in Man (OMIM) database entry on type I SMA: https://www.omim.org/entry/253300

- ClinicalTrials.gov search for SMA: https://clinicaltrials.gov/ct2/ results?cond=spinal+muscular+atrophy\&term $=\&$ cntry $1=$

\&state $1=\&$ SearchAll=Search+all+studies\&recrs

- U.S. Food \& Drug Administration (FDA) Vaccines, Blood and Biologics section: https://www.fda.gov/BiologicsBloodVaccines/ default.htm

- European Medicines Agency (EMA) Advanced Therapy Medicinal Products (ATMPs): http://www.ema.europa.eu/ema/index. jsp?curl=pages/regulation/general/general_content_000294. jsp\&mid=WC0b01ac05800241e0

- China Food and Drug Administration (SFDA): http://eng.sfda. gov.cn/WS03/CL0755

- SMA News Today: https://smanewstoday.com

- Cure SMA (USA): http://www.curesma.org

- SMA Foundation (USA): http://www.smafoundation.org

- SMA Europe: http://www.sma-europe.eu

- SMA Support UK: http://www.smasupportuk.org.uk

- The SMA Trust (UK): http://www.smatrust.org
- Association Française contre les Myopathies (AFM, France): http://www.afm-telethon.fr

- Deutsche Gesellschaft fuer Muskelkranke/Initiative SMA (DGM, Germany): http://www.initiative-sma.de

- Famiglie SMA (Italy): http://www.famigliesma.org

- Fundacja SMA (Poland): http://www.fsma.pl

- Prinses Beatrix Spierfonds (Netherlands): https://prinsesbeatrix spierfonds.nl

- Vereniging Spierziekten Nederland (VSN, Netherlands): http:// www.spierziekten.nl

- Fundación AME (FundAME, Spain): http://www.fundame.net

- Familias AME Argentina: http://www.fameargentina.com.ar

- SMA Australia: https://smaaustralia.org.au

- Families of SMA Hong Kong: http://www.fsma.org.hk

- Children with SMA (Ukraine): http://www.csma.org.ua

- Genetic Alliance (USA): http://www.geneticalliance.org

- Rare Diseases Europe (EURORDIS): http://www.eurordis.org

- Genetic Alliance UK: https://www.geneticalliance.org.uk

- Findacure: http://www.findacure.org.uk

- American Society for Gene and Cell Therapy (ASGCT): http:// www.asgct.org

- European Society for Gene and Cell Therapy (ESGCT): https:// www.esgct.eu

- Australasian Gene and Cell Therapy Society (AGCTS): http://agts. org.au

- International Society for Stem Cell Research (ISCCR): http://www. isscr.org

- International Rare Diseases Research Consortium (IRDiRC): http://www.irdirc.org

- Treat NMD (Europe): http://www.treat-nmd.eu/

\section{CONFLICT OF INTEREST}

RJY-M is a member of the UK SMA Research Consortium and receives research funding for his laboratory from The SMA Trust through the consortium.

V Prakash and RJ Yáñez-Muñoz AGCTlab.org, Centre for Biomedical Sciences, School of Biological Sciences, Royal Holloway, University of London, Egham, UK E-mail: rafael.yanez@royalholloway.ac.uk or ryanez-gteditor@royalholloway.ac.uk 